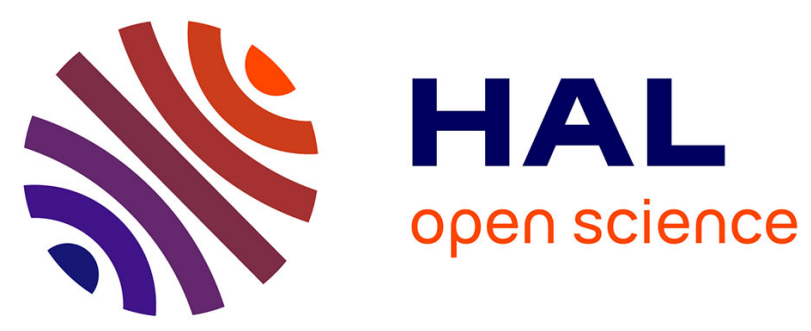

\title{
Shape coexistence in light krypton isotopes
}

E. Clément, A. Görgen, E. Bouchez, A. Chatillon, W. Korten, Y. Le Coz, C. Theisen, C. Andreoiu, F. Becker, Bertram Blank, et al.

\section{To cite this version:}

E. Clément, A. Görgen, E. Bouchez, A. Chatillon, W. Korten, et al.. Shape coexistence in light krypton isotopes. Conference on Nuclei at the Limits, Jul 2004, Argonne, Illinois, United States. pp.55-61, 10.1063/1.1905291 . in2p3-00024208

\section{HAL Id: in2p3-00024208 https://hal.in2p3.fr/in2p3-00024208}

Submitted on 8 Jan 2007

HAL is a multi-disciplinary open access archive for the deposit and dissemination of scientific research documents, whether they are published or not. The documents may come from teaching and research institutions in France or abroad, or from public or private research centers.
L'archive ouverte pluridisciplinaire HAL, est destinée au dépôt et à la diffusion de documents scientifiques de niveau recherche, publiés ou non, émanant des établissements d'enseignement et de recherche français ou étrangers, des laboratoires publics ou privés. 


\title{
Shape coexistence in light Krypton isotopes
}

\author{
E. Clément*, A. Görgen*, E. Bouchez*, A. Chatillon*, W. Korten*, Y. Le Coz*, \\ Ch. Theisen*, C. Andreoiu ${ }^{\dagger}$, F. Becker**, B. Blank ${ }^{\ddagger}$, C. Borcea ${ }^{\S}$, A. Buta ${ }^{\S}$, \\ P. Butler ${ }^{\dagger}$, J.M. Casandjian ${ }^{\mathbb{I}}$, W.N. Catford ${ }^{\|}$, T. Czosnyka ${ }^{\dagger \dagger}$, A. Emsallem ${ }^{\ddagger}$, G. de \\ France $^{\mathbb{I l}}$, J. Genevey ${ }^{\S \S}$, J. Gerl ${ }^{* *}$, F. Hannachi ${ }^{\ddagger}$, K. Hauschild ${ }^{\text {IIII }}$, R.-D. Herzberg ${ }^{\dagger}$, \\ A. Hürstel ${ }^{*}$, J. Iwanicki ${ }^{\dagger}$, D. Jenkins ${ }^{\dagger}$, G. Jones ${ }^{\dagger}$, M. Lewitowicz ${ }^{\mathbb{I}}$, R. Lucas*, \\ I. Matea ${ }^{\text {II }}$, F. Negoita ${ }^{\S}$, F. de Oliviera Santos ${ }^{\text {II }}$, D. Pantelica ${ }^{\S}$, J. Pinston ${ }^{\S \S}$, \\ P. Rahkila***, M. Rejmund ${ }^{\mathbb{I}}$, G. Sletten ${ }^{\dagger \dagger \dagger}$, M. Stanoiu ${ }^{\mathbb{I}}$, C. Timis ${ }^{\|}$, R. Wadsworth ${ }^{\ddagger \ddagger}$, \\ J.N. Wilson* and M. Zielińska ${ }^{\dagger \dagger}$ \\ ${ }^{*}$ DAPNIA/SPhN, CEA Saclay, F-91191 Gif-sur-Yvette, France \\ Dept. of Physics, University of Liverpool, Liverpool L69 7ZE, UK \\ ${ }^{* *}$ Gesellschaft für Schwerionenforschung, D-64291 Darmstadt, Germany \\ ${ }^{\ddagger}$ CEN Bordeaux-Gradignan, IN2P3-CNRS, F-33175 Gradignan Cedex, France \\ ${ }^{\S}$ IFIN-HH, P.O. Box MG6, ROM-76900 Bucarest-Magurele, Romania \\ ${ }^{\mathbb{}}$ GANIL, F-14076 Caen Cedex, France \\ "Dept. of Physics, University of Surrey, Guildford GU2 7XH, UK \\ ${ }^{\dagger}$ Heavy Ion Laboratory, Warsaw University, PL-02097 Poland \\ \#IPN Lyon, IN2P3-CNRS, F-68622 Villeurbanne Cedex, France \\ ${ }^{\$} I S N$, IN2P3-CNRS, F-38026 Grenoble, France \\ III CSNSM, IN2P3-CNRS, F-91405 Orsay, France \\ *** Dept. of Physics, University of Jyväskylä, FIN-40014 Jyväskylä, Finland \\ ${ }^{\dagger \dagger}$ Niels Bohr Institute, Belgdamsvej 17, DK-2100 Copenhagen Ø, Denmark \\ HDDept. of Physics, University of York, Heslington, York YO10 5DD, UK
}

\begin{abstract}
Shape coexistence in the light krypton isotopes was studied in a series of experiments at GANIL using various experimental techniques. A new low-lying $0^{+}$state, a so-called shape isomer, was found in delayed conversion-electron spectroscopy after fragmentation reactions. The systematics of such low-lying $0^{+}$states suggests that the ground states of the isotopes ${ }^{78} \mathrm{Kr}$ and ${ }^{76} \mathrm{Kr}$ have prolate deformation, while states with prolate and oblate shape are practically degenerate and strongly mixed in ${ }^{74} \mathrm{Kr}$, and that the oblate configuration becomes the ground state in ${ }^{72} \mathrm{Kr}$. This scenario was tested in experiments performing low-energy Coulomb excitation of radioactive ${ }^{76} \mathrm{Kr}$ and ${ }^{74} \mathrm{Kr}$ beams from the SPIRAL facility. Both transitional and diagonal electromagnetic matrix elements were extracted from the observed $\gamma$-ray yields. The results find the prolate shape for the ground-state bands in ${ }^{76} \mathrm{Kr}$ and ${ }^{74} \mathrm{Kr}$ and an oblate deformation for the excited $2_{2}^{+}$state in ${ }^{74} \mathrm{Kr}$, confirming the proposed scenario of shape coexistence.
\end{abstract}

\section{Introduction}

Nuclei in-between closed shells generally exhibit a deformation of their ground states, which is governed by a delicate interplay between microscopic and macroscopic effects. Elongated (prolate) ground-state shapes are found far more abundantly than compressed (oblate) deformations. Of particular interest in this context is the $A \sim 70$ region around selenium $(\mathrm{Z}=34)$ and krypton $(\mathrm{Z}=36)$ isotopes near the $N=Z$ line, in which oblate ground-state configurations are predicted. This can be understood qualitatively from the single-particle level scheme, where pronounced gaps are found at large values of both prolate and oblate deformations for proton and neutron numbers 34 and 36 . As a consequence of this competition of shell gaps, states with configurations based on different deformations coexist within a narrow energy range. This phenomenon is called shape coexistence. Many calculations have been performed 
in the Se-Kr region using various mean-field approaches [1, 2, 3]. All these studies find oblate and prolate shapes competing in these nuclei. However, they predict significant differences in the excitation energy of shape-coexisting states and in their decay properties.

An experimental fingerprint of shape coexistence in even-even nuclei is the observation of low-lying excited $0^{+}$ states. Such a state can be understood as the "ground state" of a different shape. If two such $0^{+}$states are close in energy, one would expect a strong mixing of the different configurations. If in addition to that the difference in deformation is large, e.g. in the case of oblate-prolate shape coexistence, the electric monopole (E0) strength $\rho^{2}(E 0)$ for transitions between such states becomes large. Electric monopole transitions are non-radiative and can only proceed via internal conversion or internal pair creation (if the energy is above the rest mass of the electron-positron pair). If the excited $0_{2}^{+}$state is near or even below the $2_{1}^{+}$state, it decays predominantly or even exclusively via the E0 transition to the ground state. In these cases the excited $0_{2}^{+}$state becomes a metastable shape isomer.

An experiment to search for such shape isomers in the light krypton isotopes is discussed in the first section. The results support a shape coexistence scenario with an inversion of the ground-state shape from prolate to oblate going from ${ }^{76} \mathrm{Kr}$ to ${ }^{72} \mathrm{Kr}$ and almost degenerate unperturbed prolate and oblate states in ${ }^{74} \mathrm{Kr}$. While this interpretation is very conclusive, it is only based on indirect observations. In order to prove the scenario, direct measurements of the shapes have been performed using Coulomb excitation of low-energy radioactive beams and the reorientation effect [4]. These experiments are discussed in the second section.

\section{Shape isomers in the light krypton isotopes}

Low-lying $0_{2}^{+}$states were known in the light even-even $\mathrm{Kr}$ isotopes with $N>Z$. The relevant parts of the level schemes are shown in Fig. 1. The excitation energy of the $0_{2}^{+}$states is decreasing going from ${ }^{78} \mathrm{Kr}$ to ${ }^{74} \mathrm{Kr}$, where it is very close to the $2_{1}^{+}$state. While these states decay predominantly via E2 transitions to the $2^{+}$states in the heavier isotopes, the direct E0 decay to the ground state becomes competitive to the highly converted low-energy transition to the $2_{1}^{+}$in ${ }^{74} \mathrm{Kr}$. This results in a relatively long lifetime of $\sim 20 \mathrm{~ns}$ for the $0_{2}^{+}[5,6]$. A search for such isomers was performed at GANIL and revealed a new $0^{+}$isomer in ${ }^{72} \mathrm{Kr}$ and confirmed the results for ${ }^{74} \mathrm{Kr}$ [7].

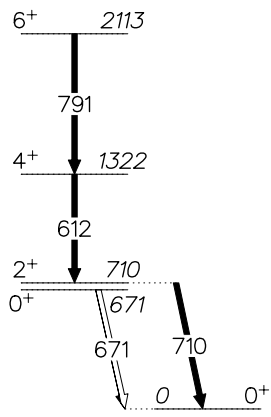

${ }^{72} \mathrm{Kr}$

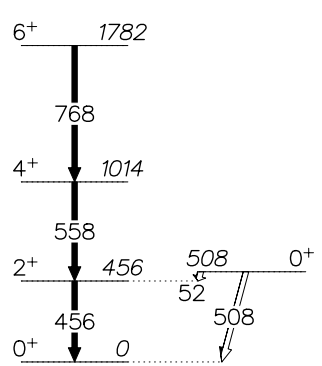

${ }^{74} \mathrm{Kr}$

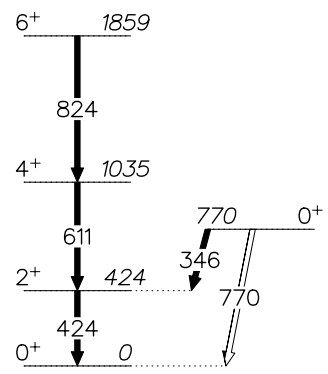

${ }^{76} \mathrm{Kr}$

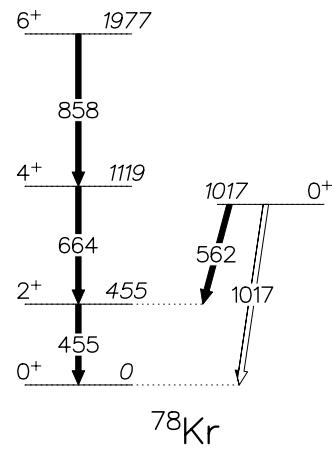

FIGURE 1. Partial level schemes of the even-even $\mathrm{Kr}$ isotopes from ${ }^{72} \mathrm{Kr}$ to ${ }^{78} \mathrm{Kr}$ illustrating the systematics of excited $0^{+}$states. States with proposed prolate configuration are shown on the left, those with proposed oblate configuration on the right of each level scheme. Transitions proceeding via conversion electrons are shown as open arrows.

A primary beam of ${ }^{78} \mathrm{Kr}$ was accelerated to $73 \mathrm{MeV} / \mathrm{A}$ in the GANIL cyclotrons and then fragmented on a Be target of $530 \mu \mathrm{m}$ thickness. The primary beam intensity was $\sim 20 \mathrm{pnA}$. The fragments were separated in the LISE3 spectrometer [8] consisting of two dipole magnets with a degrader wedge in between, and a Wien velocity filter. The intensity of the fragments was $\sim 240$ ions/s for ${ }^{74} \mathrm{Kr}$ and 3 ions $/ \mathrm{s}$ for ${ }^{72} \mathrm{Kr}$. The ions were identified event-by-event by measuring the time of flight and the energy loss in a stack of three silicon detectors. These detectors were used at the same time to slow down the ions in order to implant them into a Kapton catcher foil of $25 \mu \mathrm{m}$ thickness. Subsequent isomeric decays were recorded in a liquid-nitrogen cooled $\mathrm{Si}(\mathrm{Li})$ detector for conversion electrons and two EXOGAM Clover and a LEPS detector for $\gamma$ rays.

The spectrum of the $\mathrm{Si}(\mathrm{Li})$ detector in coincidence with ions identified as ${ }^{72} \mathrm{Kr}$ is shown in Fig. 2. Two peaks are present corresponding to the $K$ and $L$ conversion lines of a $671 \mathrm{keV}$ transition. The corresponding $\gamma$-ray spectra from 


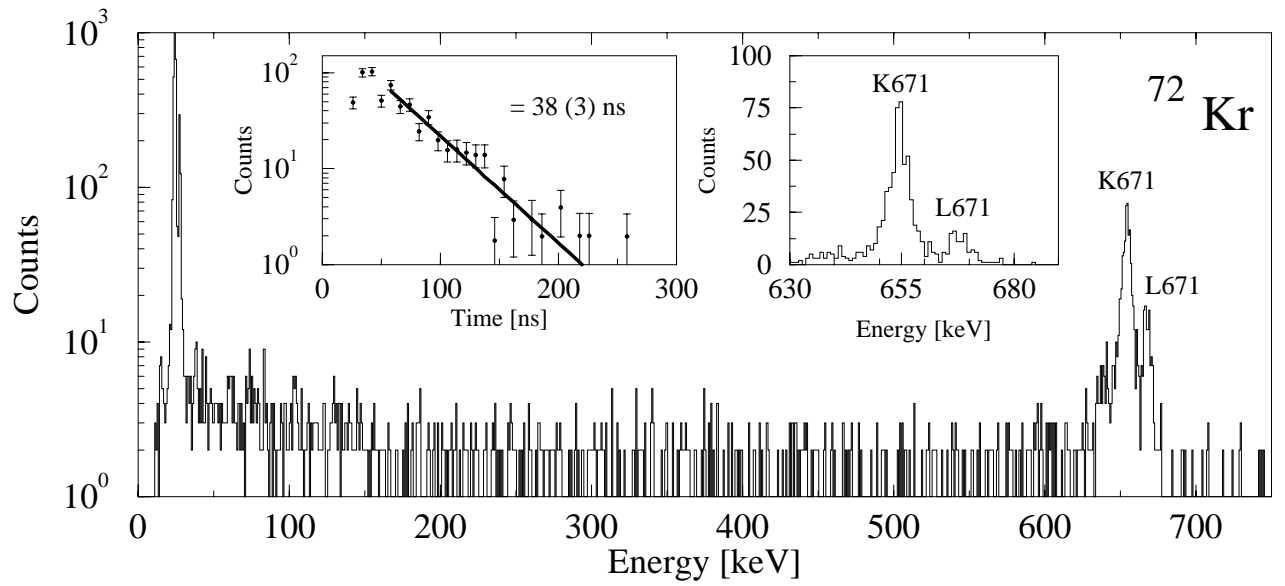

FIGURE 2. Conversion-electron spectrum in coincidence with ${ }^{72} \mathrm{Kr}$ fragments. The insets show the time spectrum of the electrons and the energy spectrum on a linear scale, respectively. The figure is taken from ref. [7]

the Ge detectors do not show any lines, suggesting E0 character of the transition, which is confirmed by the intensity ratio of the $K$ and $L$ lines. Thus, a new $0^{+}$state is placed at $671 \mathrm{keV}$ excitation energy, just below the first $2^{+}$state at $710 \mathrm{keV}$.

The ions are fully stripped of their atomic electrons when passing through the spectrometer. Since conversionelectron decay is the only decay mode of the $0_{2}^{+}$state, the nucleus is trapped in this state and can only decay after it was slowed down in the stack of Si detectors and finally stopped in the Kapton foil, regaining atomic electrons in the process. Therefore the lifetime has to be measured against the signal coming from the last Si detector in which the ions are slowed down, and it can be much shorter than the flight time through the spectrometer, which is of the order of $\sim 400 \mathrm{~ns}$. The lifetime of the $0_{2}^{+}$state was in this way measured to be 38(3) ns. This results in an electric monopole strength of $\rho^{2}(E 0)=(72 \pm 6) \times 10^{-3}$, which is among the largest E0 strengths to be found in nuclei. The lifetime of the $0_{2}^{+}$state in ${ }^{74} \mathrm{Kr}$ was measured to be 19(2) ns, in agreement with the earlier measurements. Taking into account the branching ratio of the $\mathrm{E} 0$ transition and the decay to the $2_{1}^{+}$state, this results in an even bigger electric monopole strength of $\rho^{2}(E 0)=(85 \pm 19) \times 10^{-3}$ for ${ }^{74} \mathrm{Kr}$.

The new shape isomer completes the systematic of low-lying excited $0^{+}$states in the $\mathrm{Kr}$ isotopes down to the $N=Z$ line, as is illustrated in Fig. 1. The excitation energy of the $0_{2}^{+}$states reaches a minimum in ${ }^{74} \mathrm{Kr}$ and is increasing again in ${ }^{72} \mathrm{Kr}$. All isotopes show a regular rotational behavior at high spin and a perturbation at low spin, indicating a mixing of different configurations. It can be assumed that the configurations are pure at high spin, where the bands are regular, so that the energies of the pure low-spin states can be extrapolated. The energy difference of the extrapolated pure states and the observed mixed states can be used to derive the interaction strength and the mixing amplitudes for the assumed prolate and oblate configurations. It is found that the mixing is increasing going from ${ }^{78} \mathrm{Kr}$ to ${ }^{74} \mathrm{Kr}$, where the pure states are practically degenerate and the observed energy difference of $\sim 500 \mathrm{keV}$ is entirely due to the maximal mixing of the configurations. For ${ }^{72} \mathrm{Kr}$ the mixing is reduced again, which together with the parabolic behavior of the $0_{2}^{+}$states can be interpreted as an inversion of the ground-state shape. While the ground states are assumed to be prolate in the heavier isotopes, the oblate configuration is coming down in energy, crossing over in ${ }^{74} \mathrm{Kr}$, and becoming the new ground-state configuration in ${ }^{72} \mathrm{Kr}$, while the new shape isomer is believed to be the band head of the known prolate rotational band.

It was the aim of the Coulomb excitation experiments, which can give information on the intrinsic shape of the nuclei, to prove this scenario of shape coexistence in the light $\mathrm{Kr}$ isotopes.

\section{Coulomb excitation of ${ }^{76} \mathrm{Kr}$ and ${ }^{74} \mathrm{Kr}$ SPIRAL beams}

Coulomb excitation is a well-established technique to populate collective states that are linked to the ground state by E2 matrix elements. At energies well below the Coulomb barrier it is a pure electromagnetic process and the interaction time between the projectile and the target is long enough to populate higher-lying states in a multiple-step process. The electromagnetic interaction can be calculated with high precision, including higher-order effects like the so-called 
reorientation effect [4] which causes a difference in the differential Coulomb excitation cross section for different intrinsic shapes of the nuclei. By measuring the excitation probability as a function of the scattering angle and/or as a function of the atomic number $Z$, diagonal matrix elements, and therefore the intrinsic shape, can be extracted, if the the precision of the measurement is high enough.

A primary ${ }^{78} \mathrm{Kr}$ beam of $\sim 10^{12} \mathrm{pps}$ was accelerated in the GANIL cyclotrons to an energy of $68.5 \mathrm{MeV} / \mathrm{A}$ and then fragmented on a carbon target of the SPIRAL facility. The radioactive ions were extracted using the ISOL method and then re-accelerated in the compact CIME cyclotron to energies below the coulomb barrier. A secondary beam intensity of $5 \times 10^{5}$ pps for ${ }^{76} \mathrm{Kr}$ and $10^{4}$ pps for ${ }^{74} \mathrm{Kr}$ was reached. The projectiles were Coulomb excited on ${ }^{208} \mathrm{~Pb}$ targets of $\sim 1 \mathrm{mg} / \mathrm{cm}^{2}$ thickness at $4.4 \mathrm{MeV} / \mathrm{A}\left({ }^{76} \mathrm{Kr}\right)$ and $4.7 \mathrm{MeV} / \mathrm{A}\left({ }^{74} \mathrm{Kr}\right)$. In the ${ }^{76} \mathrm{Kr}$ case also a ${ }^{48} \mathrm{Ti}$ target was used with a beam energy of $2.6 \mathrm{MeV} / \mathrm{A}$. An annular double-sided silicon detector segmented into 16 concentric rings and 16 sectors was used to detect either the scattered projectiles or the recoiling target nuclei. The energy resolution of the silicon detector and the kinematics of the reaction allowed to distinguish between the $\mathrm{Kr}$ and $\mathrm{Pb}$ nuclei detected in the $\mathrm{Si}$ detector. The distance between the target and the detector was chosen such that a continuous range of scattering angles between $18^{\circ} \leq \theta_{C M} \leq 128^{\circ}$ in the center-of-mass frame was covered by either detecting the projectile or the target nucleus. Gamma-rays were detected in the EXOGAM array, which comprised $7\left({ }^{76} \mathrm{Kr}\right)$ and $11\left({ }^{74} \mathrm{Kr}\right)$ Clover detectors at the time of the experiments, respectively. The emission angle of the $\gamma$ rays relative to the direction of the scattered projectile was measured with good precision due to the segmentation of both the silicon and the germanium detectors. This reduced the Doppler broadening of the peaks to $8 \mathrm{keV}$ for transitions of $500 \mathrm{keV}$.

In both the ${ }^{76} \mathrm{Kr}$ and the ${ }^{74} \mathrm{Kr}$ experiments, states up to the $8^{+}$were populated in the ground-state bands, and several transitions connecting non-yrast states were observed. All transitions that were observed are shown in the level schemes of Fig. 4. The total data sets were divided into four subsets corresponding to different ranges of the scattering angles. Example spectra for large and small scattering angles are shown in Fig. 3. At large scattering angles, where the impact parameter is small, there is a much higher probability to populate high-lying states in a multiple-step process. The resulting $\gamma$-ray yields for all transitions observed for the four different angular ranges were analyzed with the code GOSIA [9]. Both transitional and diagonal matrix elements are found in a $\chi^{2}$ minimization by comparing the observed $\gamma$-ray yields with the ones calculated from the matrix elements. Any known spectroscopic information such as lifetimes, mixing and branching ratios can and should be used as constraints in the minimization process.
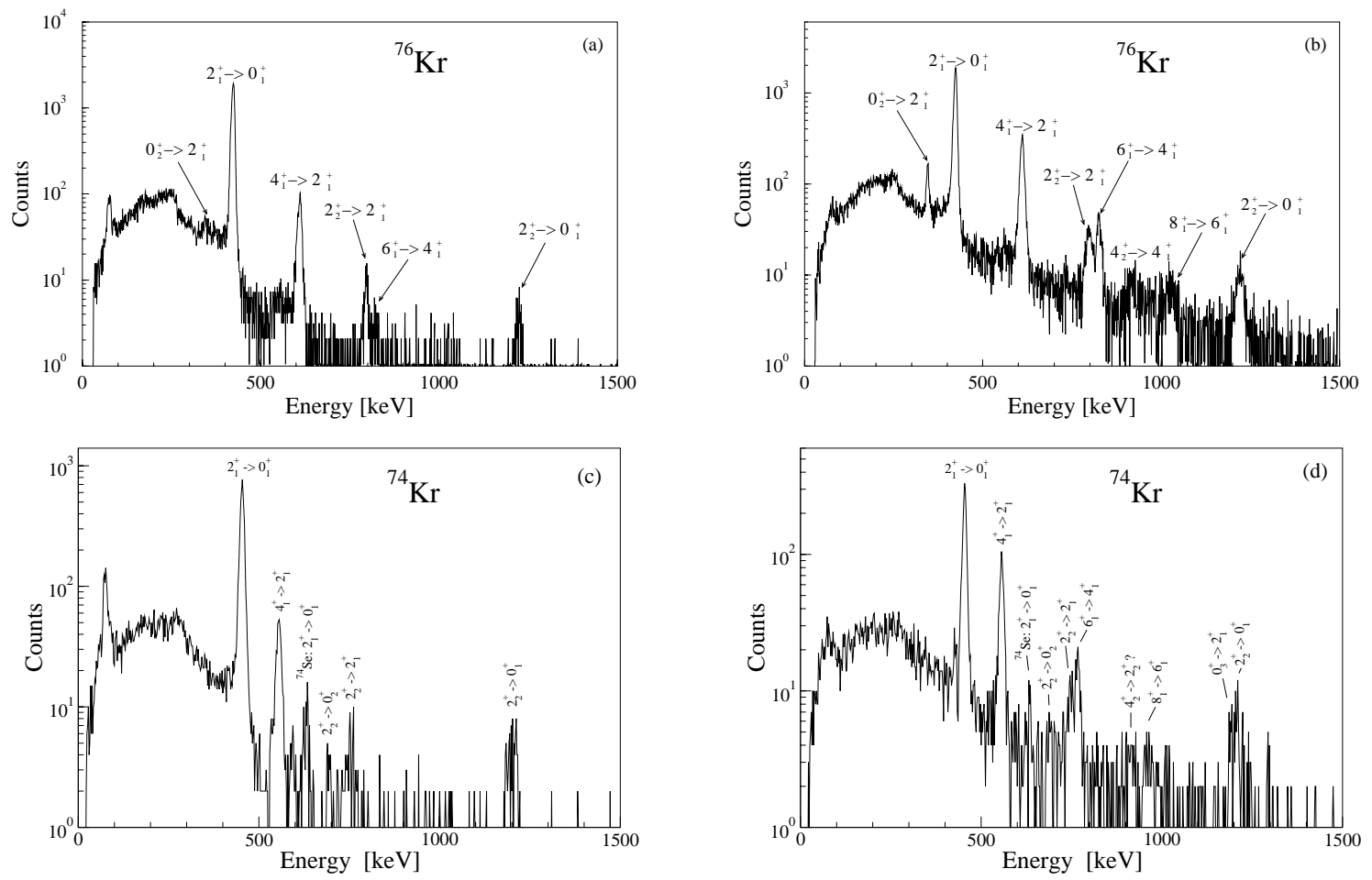

FIGURE 3. Gamma-ray spectra observed after Coulomb excitation of the ${ }^{76} \mathrm{Kr}$ and ${ }^{74} \mathrm{Kr}$ projectiles on ${ }^{208} \mathrm{~Pb}$ targets for different scattering angles (given in the center-of-mass frame) of the projectiles: (a) ${ }^{76} \mathrm{Kr}$ with $40^{\circ} \leq \theta_{C M} \leq 49^{\circ}$, (b) ${ }^{76} \mathrm{Kr}$ with $71^{\circ} \leq \theta_{C M} \leq 88^{\circ}$, (c) ${ }^{74} \mathrm{Kr}$ with $18^{\circ} \leq \theta_{C M} \leq 41^{\circ}$, (d) ${ }^{74} \mathrm{Kr}$ with $51^{\circ} \leq \theta_{C M} \leq 77^{\circ}$ 

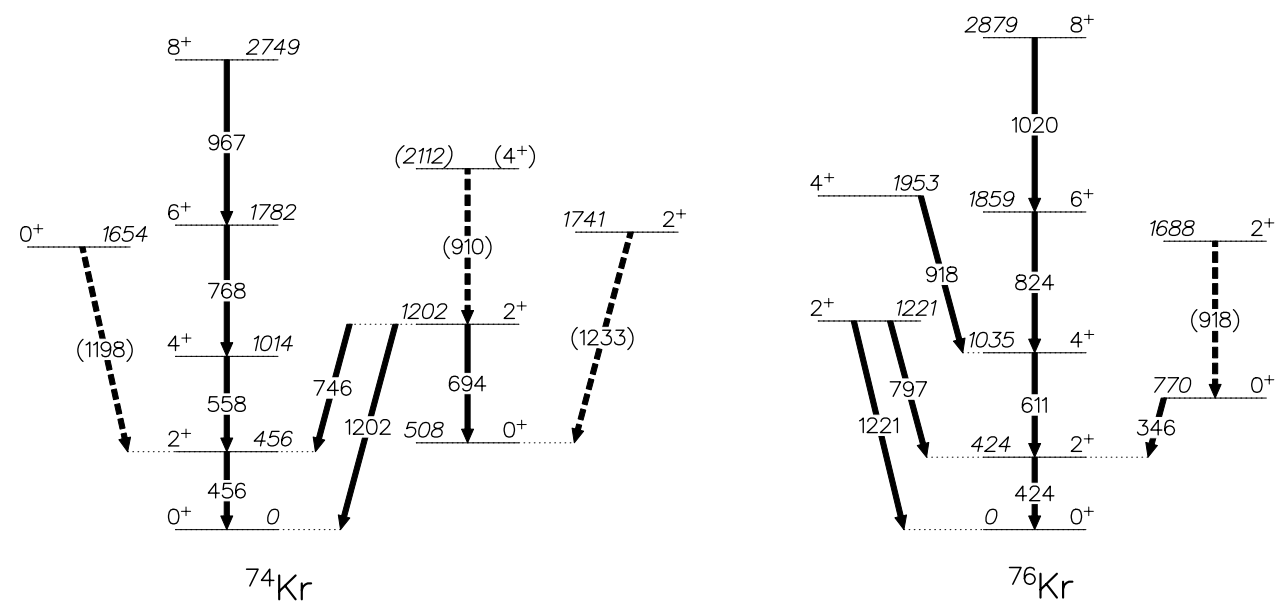

FIGURE 4. Partial level schemes of ${ }^{74} \mathrm{Kr}$ and ${ }^{76} \mathrm{Kr}$ showing the observed transitions after Coulomb excitation. Transitions that could not be identified unambiguously are shown as dashed arrows.

\section{Results for ${ }^{76} \mathrm{Kr}$}

The $\gamma$-ray yields observed at four different ranges of the center-off-mass scattering angle for the ${ }^{208} \mathrm{~Pb}$ target and at three different ranges for the ${ }^{48} \mathrm{Ti}$ target were analyzed with the code GOSIA [9]. A total of 35 matrix elements, both transitional and diagonal, enter into the calculation and are found in a least-squares fitting procedure. Known spectroscopic information such as lifetimes, branching and mixing ratios [10] are used as constraints of the fit. For the transitional quadrupole moments in the ground-state band a constant value of $2.8 \mathrm{eb}$ was found. A precision between $1 \%$ and $3 \%$ was reached for the individual values. These results confirm the $B(E 2)$ strengths known from lifetime measurements [11]. The static quadrupole moments for the states in the ground-state band were extracted from the diagonal matrix elements. The absolute values are similar to the transitional quadrupole moments and have positive sign, indicating that the deformation of the ground-state band is prolate, as expected in the shape coexistence scenario. The precision reached for the static quadrupole moments is about $10 \%$, leaving no doubt about the sign of the quadrupole moment. It was for the first time that static quadrupole moments were measured in a radioactive-beam experiment.

While transitional quadrupole moments were also found for non-yrast transitions, it was not possible to extract the diagonal matrix elements of any non-yrast states. Since the $4_{2}^{+} \rightarrow 2_{2}^{+}$transition was not observed, no diagonal matrix element was found for the $2_{2}^{+}$state. This structure is interpreted as a $\gamma$-vibrational band. The $2_{3}^{+} \rightarrow 0_{2}^{+}$transition has the same energy as the $4_{2}^{+} \rightarrow 4_{1}^{+}$transition and the states are populated only very weakly. Therefore the static quadrupole moment of the $2_{3}^{+}$state, which is believed to have an oblate configuration, could not be extracted, either.

\section{Results for ${ }^{74} \mathrm{Kr}$}

The experiment on ${ }^{74} \mathrm{Kr}$ showed that low-energy Coulomb excitation and reorientation measurements are possible with beam intensities of $10^{4}$ pps. However, the level of statistics is at the very minimum required for such a measurement. A small contamination of the isobar ${ }^{74} \mathrm{Se}$ of $\sim 2 \%$ was present in the beam, and the $2_{1}^{+} \rightarrow 0_{1}^{+}$transition of that nucleus is visible in the spectra. No sign of the $4_{1}^{+} \rightarrow 2_{1}^{+}$transition in ${ }^{74}$ Se could be found. Like in the ${ }^{76} \mathrm{Kr}$ case, the total data set was divided into four subsets corresponding to different ranges of scattering angles. It should be mentioned that the data are still under evaluation and that the results presented here are preliminary. The transitional quadrupole moments within the ground-state band could be measured with a similar precision as in the ${ }^{76} \mathrm{Kr}$ case. However, the measured lifetimes of the $2_{1}^{+}$and $4_{1}^{+}$states [12] cannot be reproduced when they are treated as free parameters in the GOSIA fit. Using these lifetime values as constraints in the fit results in a distinctly lower quadrupole moment for the $4_{1}^{+}$state compared to the other states in the band. In order to base the Coulomb excitation results on a more solid footing, a new lifetime measurement using the recoil-distance method will be performed in the near future.

As in the case of ${ }^{76} \mathrm{Kr}$, the static quadrupole moment of the $2_{1}^{+}$state has positive sign, confirming the prolate 
character of the ground-state band. The absolute value might change somewhat depending on the outcome of the new lifetime measurement, but the sign of the quadrupole moment would not change even if significantly different lifetimes were found in the new experiment. An important result with respect to the shape coexistence scenario is the static quadrupole moment for the $2_{2}^{+}$state. The value derived from the diagonal matrix element is negative, confirming the oblate shape of this structure. The absolute value of the quadrupole moment of the $22_{2}^{+}$state depends on the yield for the $4_{2}^{+} \rightarrow 2_{2}^{+}$transition. The $4_{2}^{+}$state, however, is not known. The most consistent fit of the data is achieved if the $910 \mathrm{keV}$ transition, that cannot be attributed otherwise, is assumed to be the missing $4_{2}^{+} \rightarrow 2_{2}^{+}$transition. We hope to observe this transition in the future lifetime experiment, and in addition to that to measure the lifetime of the $22_{2}^{+}$ state. A more precise value for the static quadrupole moment will be achievable with these additional constraints. The observed $\gamma$-ray yields cannot be reproduced and consistently explained if the opposite (prolate) sign is assumed for the $2_{2}^{+}$state. Even though we are not in the position to give an absolute value for the static quadrupole moment at this time without a more reliable lifetime measurement, there is no doubt about the sign of the static quadrupole moment, and therefore about the oblate shape of the $2_{2}^{+}$state.

High-lying $0_{3}^{+}$and $2_{3}^{+}$states were observed recently after $\beta$ decay [13]. Transitions depopulating these states are observed only tentatively, and no information about their structure can be extracted from the data.

\section{Summary and outlook}

A new shape isomer was observed in ${ }^{72} \mathrm{Kr}$ in conversion-electron spectroscopy after fragmentation reactions, extending the systematics of excited $0^{+}$states in the light $\mathrm{Kr}$ isotopes to the $N=Z$ line. The lifetimes of the $0_{2}^{+}$ states were measured in ${ }^{72} \mathrm{Kr}$ and ${ }^{74} \mathrm{Kr}$ and the strengths of the electric monopole transitions depopulating these states were derived. Mixing amplitudes of the prolate and oblate configurations were extracted; they suggest together with systematics of the excitation energies of the $0_{2}^{+}$states an inversion of the ground-state shapes from prolate in ${ }^{76} \mathrm{Kr}$ to oblate in ${ }^{72} \mathrm{Kr}$.

In another series of experiments, low-energy Coulomb excitation has been performed with ${ }^{76} \mathrm{Kr}$ and ${ }^{74} \mathrm{Kr}$ projectiles produced at the SPIRAL facility at GANIL. States up to the $8^{+}$and several non-yrast states were populated. Transitional and diagonal matrix elements were extracted from the $\gamma$-ray yields observed at several ranges of scattering angles. The results confirm the prolate shape for the ground-state bands in ${ }^{76} \mathrm{Kr}$ and ${ }^{74} \mathrm{Kr}$, and an oblate shape is found for the $2_{2}^{+}$state in ${ }^{74} \mathrm{Kr}$.

It would be highly desirable to study ${ }^{72} \mathrm{Kr}$ with low-energy Coulomb excitation. Unfortunately the beam intensities currently available for ${ }^{72} \mathrm{Kr}$ from SPIRAL are too low to perform such a measurement. Coulomb excitation of ${ }^{72} \mathrm{Kr}$ and ${ }^{68} \mathrm{Se}$ was recently performed at intermediate energies of $\sim 60 \mathrm{MeV} / \mathrm{A}$. This method has the advantage that very thick targets can be used, partly compensating the low beam intensity. However, only single-step excitations populating $2^{+}$states are possible due to the short interaction times. While it is not possible to measure the intrinsic shape using the reorientation effect at intermediate energies, the excited $2_{2}^{+}$states with prolate and oblate configuration can be identified and the $B(E 2)$ transition strengths can be measured. The data for ${ }^{72} \mathrm{Kr}$ and ${ }^{68}$ Se are currently under investigation.

\section{REFERENCES}

1. P. Bonche et al., Nucle. Phys. A443, 39 (1985)

2. W. Nazarewicz et al., Nucl. Phys. A435, 397 (1985)

3. A. Petrovici et al., Nucl. Phys. A483, 317 (1988)

4. J. de Boer and J. Eichler, Advances in Nucl. Phys. Vol. 1, 1 (1968)

5. C. Chandler et al., Phys. Rev. C 56, R2924 (1997); Phys. Rev. C 61, 044309 (2000)

6. F. Becker et al., Eur. Phys. J. A 4, 103 (1999)

7. E. Bouchez et al., Phys. Rev. Lett. 90, 082502 (2003)

8. R. Anne and R.C. Mueller, Nucl. Instrum. Meth. Phys. Res. B 70, 276 (1992)

9. T. Czosnyka, D. Cline, C.Y. Wu, Bull. Amer. Phys. Soc. 28, 745 (1983); GOSIA users manual, University of Rochester, UR-NSRL-305 (1991)

10. B. Singh, Nucl. Data Sheets 74, 63 (1995)

11. B. Wormann et al., Nucl. Phys. A431, 170 (1984)

12. J. Roth et al., J. Phys. G: Nucl. Phys. 10, L25 (1984)

13. A. Piechaczek et al., Phys. Rev. C 67, 051305 (2003) 